\title{
Electrochemical DNA-Based Immunoassay That Employs Steric Hindrance To Detect Small Molecules Directly in Whole Blood
}

\author{
Sahar S. Mahshid, ${ }^{\dagger}$ Francesco Ricci, ${ }^{\S}$ Shana O. Kelley, ${ }^{\ddagger}$ and Alexis Vallée-Bélisle* ${ }^{\dagger}{ }^{\dagger}$ \\ ${ }^{\dagger}$ Laboratory of Biosensors \& Nanomachines, Département de Chimie, Université de Montréal, Montreal, Québec H3T 1J4, Canada \\ ${ }^{\S}$ Dipartimento di Scienze e Tecnologie Chimiche, University of Rome Tor Vergata, Via della Ricerca Scientifica, Rome 00133, Italy \\ ${ }^{\ddagger}$ Department of Pharmaceutical Science, Leslie Dan Faculty of Pharmacy, University of Toronto, Toronto, Ontario M5S 3M2, \\ Canada
}

\section{Supporting Information}

ABSTRACT: The development of a universal sensing mechanism for the rapid and quantitative detection of small molecules directly in whole blood would drastically impact global health by enabling disease diagnostics, monitoring, and treatment at home. We have previously shown that hybridization between a free DNA strand and its complementary surface-bound strand can be sterically hindered when the former is bound to large antibodies. Here, we exploit this effect to design a competitive antibody-based electrochemical assay, called CeSHHA, that enables the quantitative detection of small molecules directly in complex matrices, such as whole blood or soil. We discuss the importance of this inexpensive assay for point-of-care diagnosis and for treatment monitoring applications.
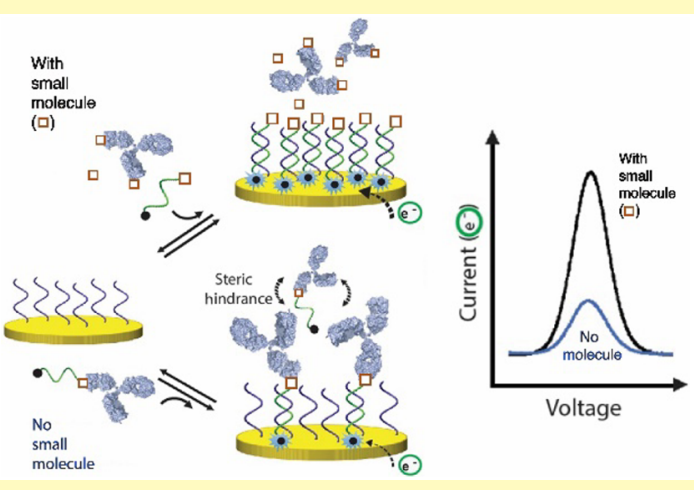

KEYWORDS: biosensors, point-of-care, DNA nanotechnology, whole blood, therapeutic drug monitoring

$\mathrm{T}$ he development of rapid, inexpensive, one-step assays for the quantitative detection of biomarkers in whole blood would drastically impact global health by enabling disease diagnosis, monitoring, and treatment at home. ${ }^{1-6}$ Enzymebased home glucometers best exemplify such technological advancements by helping millions of diabetic patients to monitor their glucose level and treat diabetes in the comfort of their home. ${ }^{7-9}$ The ability to readily detect other small molecules at home, such as amino acids, lipids, sugars, or various therapeutic drugs, would open key avenues in diagnostics and treatment for various diseases and disorders, ${ }^{10,11}$ including heart disease, ${ }^{12}$ cancers, ${ }^{13,14}$ and neurodegenerative diseases, ${ }^{15}$ such as multiple sclerosis, ${ }^{16}$ amyotrophic lateral sclerosis, ${ }^{17,18}$ Alzheimer's, and Parkinson's. ${ }^{11}$ However, the main challenges that have slowed down the commercialization of new quantitative home meters are that enzyme-based sensing mechanisms are hardly adaptable for the detection of most small molecules ${ }^{19}$ while other assays based on electrocatalysis ${ }^{20-24}$ or biorecognition ${ }^{25-28}$ typically fail when performed directly in whole blood (e.g., sensor drift due to biofouling ${ }^{27,28}$ ), or remain too cumbersome to be employed at home.

We have recently reported a highly selective electrochemical DNA-based assay, called eSHHA (for electrochemical steric hindrance hybridization assay), that enables detection of large proteins and macromolecules $(>30 \mathrm{kDa})$ through a variation of hybridization efficiencies between a signaling DNA and a surface-bound complementary capturing DNA through steric hindrance. $^{29}$ The main advantage of this novel signaling mechanism is that it remains selective enough to be employed directly in complex samples such as whole blood and remains insensitive to nonspecific adsorption of proteins on the sensor surface. In a manner analogous to the popular but cumbersome ELISA (enzyme-linked immunosorbent assay ${ }^{30,31}$ ), this electrochemical assay could, in principle, be adapted into a competitive format for the detection of a wide range of molecules directly in whole blood. In addition of enabling detection of a wide variety of molecules with a mass under 30 $\mathrm{kDa}$, this competitive assay also provides a signal-on mechanism (in contrast to the signal-off mechanism of $\mathrm{eSHHA}^{29}$ ). Here, we report CeSHHA, a competitive eSHHA, and show that this assay enables the rapid (less than $20 \mathrm{~min}$ ), inexpensive, quantitative detection of small molecules directly in complex sample matrix such as whole blood.

CeSHHA relies on the ability of DNA strands to hybridize in a very specific and selective manner to a surface-bound complementary DNA strand directly in complex matrices, with a hybridization efficiency that can be modulated by steric hindrance. More specifically, we have recently demonstrated that when a large macromolecule such as an antibody (i.e., large relative to the "small" $2.4 \mathrm{~nm}$ diameter of the DNA double helix) binds to a DNA strand, this reduces the number of DNA

Received: March 21, 2017

Accepted: $\quad$ May 8, 2017

Published: May 8, 2017 
strands that can reach and hybridize to the complementary strand attached to a surface at high density (Figure 1, bottom).

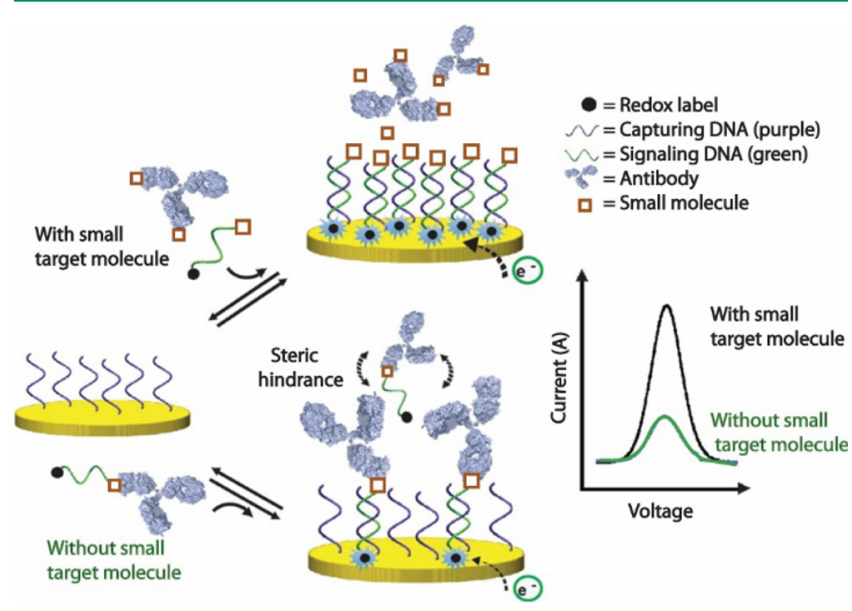

Figure 1. Schematic representation of CeSHHA, a competitive electrochemical steric hindrance hybridization immunoassay to detect small molecules. The proposed sensor is composed of densely packed electrode-bound "capturing" DNA strands (purple), free complementary signaling DNA strands (green) dual-labeled with the target small analyte $(\square)$ and a signaling redox label (black circle: methylene blue), and an antibody specific for the target molecule. In the absence of a specific analyte in the sample (bottom), the signaling strands remain bound to the antibody, which significantly reduces the ability of this large DNA complex to hybridize on the electrode surface due to steric hindrance. This ultimately generates a low electrochemical signal (blue curve). In the presence of target analytes, these later bind to the antibody and the small signaling DNA is able to reach the electrode surface and hybridize efficiently to most complementary capturing strands, thus generating a large electrochemical current (black curve) by bringing redox labels near the electrode's surface.

Such steric hindrance effects can be readily detected using electrochemical readout strategies through attachment of a redox-label molecule at one end of a "signaling" DNA strand and a small antibody-binding molecule at the other end (e.g., a hapten or epitope), and by attaching the complementary "capturing" DNA strand on a gold electrode at high surface density (Figure 1). We propose here to utilize this system to detect and quantify the concentration of small target molecules directly in complex matrices using antibodies. An antibody that specifically recognizes a small molecule is added to the sample. In the absence of a small molecule, the antibody will remain available to bind an electro-active signaling DNA strand that is conjugated with a copy of the target molecule. This will reduce the hybridization efficiency of this DNA to the electrode due to steric hindrance, thus generating a low electrochemical signal. In the presence of target molecules in the sample, the specific antibody will bind these target molecules, thus leaving the electro-active signaling DNA strands unbound and free to efficiently hybridize to the complementary capturing strands on the gold surface, generating a large electrochemical current (Figure 1). The resultant electrochemical current should be proportional to the concentration of the target molecule in solution.

To validate this novel sensing mechanism, we engineered a CeSHHA for monitoring the concentration of digoxin, a widely used drug for the treatment of heart failure and arrhythmia that displays a narrow therapeutic range $(0.5-2.0 \mathrm{ng} / \mathrm{mL}$ or $0.65-$ $2.5 \mathrm{nM}) .^{32-34}$ Due to its toxicity, digoxin uptake must be carefully monitored during patient treatment to ensure high efficiency and low toxicity. ${ }^{33,35}$ We first designed a 16-base signaling DNA strand labeled at the $3^{\prime}$ extremity with the redox-label methylene blue and at the $5^{\prime}$ end with digoxigenin, an analogue of digoxin, which is also known to bind the antibody with a poorer affinity than digoxin (see Materials and Methods in Supporting Information). This digoxin analogue allows capture of the anti-digoxin antibody without competing too strongly with the free digoxin for the binding to the antibody, which would reduce the sensitivity of the assay. We then immobilized, at high surface coverage, a 5 '-thiol-modified complementary 16-base capturing DNA strand onto the gold surface of disposable inexpensive screen printed electrodes, SPEs, via the formation of sulfur-gold bond, and backfilled with mercaptohexanol to avoid any bare portion on the gold surface.

A series of experiments were first conducted in order to determine the optimal density of the capturing strand and the optimal concentration of the signaling strand, which can be used in the smallest convenient sample volume (Figures S1, S2, S3). In brief, we found that the optimal "steric hindrance" effect is obtained at maximal surface density (surface coverage near $1.4 \times 10^{12}$ DNA strands $\left./ \mathrm{cm}^{2}\right) .{ }^{29}$ This surface coverage can be achieved on our SPE when functionalizing the electrode with $100 \mathrm{nM}$ of capturing strands overnight at room temperature (Figure S1). We then determined the optimal concentration of signaling strand to be used by performing dose-response curves of these DNA-functionalized electrodes by adding increasing concentrations of signaling strands. These experiments were performed in a $100 \mu \mathrm{L}$ sample volume that represents the minimum volume needed to cover the surface of working/counter/reference screen printed electrodes (SPE) (Figure S2). We found that $100 \mathrm{nM}$ of signaling strands is enough to saturate all capturing strands on the surface of the electrode, which generates electrochemical currents in the low microampers at the voltage expected for methylene blue $(-0.25$ $\mathrm{V}$ vs SCE). ${ }^{36}$ Finally, we also determined the minimal antibody concentration required to saturate the signaling DNA and achieve the lowest background electrochemical current. We found that an antiserum dilution of 1/6.6 (using a polyclonal anti-digoxin antibody) was sufficient to saturate the signaling strands and thus minimize the sensor background in the absence of free digoxin (Figure S4). These conditions should, in principle, ensure that our sensor displays high signal gains with detection limits in the low nanomolar range.

We then tested the response of CeSHHA for the detection of digoxin (Figure 2A). This test was performed by first adding the antiserum to the sample (1/6.6 dilution; 5 min incubation) followed by the addition of the signaling strands (100 nM; 10 min incubation). The sample is then added on the electrode and the electrochemical signal is measured using squarewave voltammetry (see Materials and Methods in Supporting Information). We first compared the hybridization efficiency (Figure 2B), and the hybridization rates (Figure 2C) of our signaling DNA strands in samples containing either no digoxin, $300 \mathrm{nM}$ digoxin, or using a signaling strand lacking the digoxigenin moiety ( $\mathrm{Ctrl}$ ). In the absence of target digoxin (Figures $2 \mathrm{~B}$, blue line), the sensor produces a small Faradaic current, consistent with the fact that the signaling strand can freely bind the antibody. The so-formed DNA-antibody complex thus creates strong steric hindrance near the electrode surface which results in a slower and less efficient hybridization (Figures $2 \mathrm{C}$, blue line $-t_{1 / 2}{ }^{\text {no digoxin }}=33 \mathrm{~min}$ ). In the presence of target 


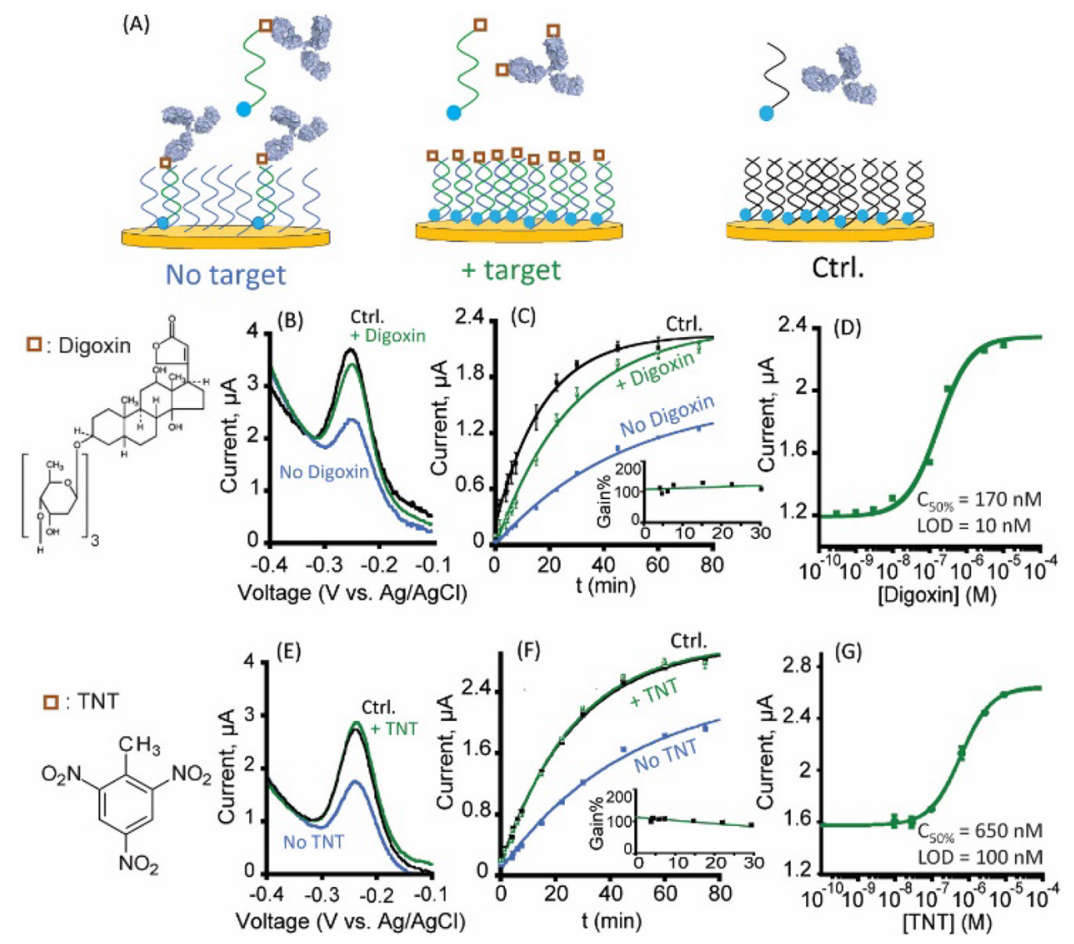

Figure 2. (A) Electrochemical detection of digoxin (B,C) and TNT (E,F), using CeSHHA. In the presence of the target molecule (300 nM digoxin and $700 \mathrm{nM}$ TNT, green line) and their respective antibodies, the small signaling strands (i.e., not bound to the antibody) are free to efficiently hybridize to the electrode-bound capturing strand, thus generating a high electrochemical current signal similar to that of the control experiment performed using a signaling strand unlabeled with digoxigenin (black line). (C,F) Hybridization is also faster in the presence of free digoxin $\left(t_{1 / 2}{ }^{\text {no }- \text { digoxin }}=33 \mathrm{~min}\right.$ and $\left.t_{1 / 2}{ }^{\text {+digoxin }}=19 \mathrm{~min}\right)$ and TNT $\left(t_{1 / 2}{ }_{-}{ }^{\text {no }}{ }^{\text {TNT }}=33 \mathrm{~min}\right.$ and $\left.t_{1 / 2}{ }^{\text {TNT }}=18 \mathrm{~min}\right)$ and the maximum signal gain $(\sim 100 \%)$ is achieved after only 5 min (insets). (D,G) Dose-response curve of digoxin (D) and TNT (G). The error bars represent the typical standard deviation obtained with three electrodes, and are dominated by interelectrode variability. $\mathrm{SWV}$ measurements were realized in $50 \mathrm{mM} \mathrm{NaH} \mathrm{PO}_{4}$, $150 \mathrm{mM} \mathrm{NaCl}, \mathrm{pH}$ 7.0. Panels $\mathrm{B}$ and $\mathrm{E}$ were obtained after $30 \mathrm{~min}$ acquisition.

digoxin at $300 \mathrm{nM}$ (Figure 2B, green line), the sensor produces almost twice the electrochemical signal in the same time-lapse (30 $\mathrm{min})$, which is consistent with the fact that free digoxin molecules sequester the antibody and thus prevent its association to the signaling strands. The hybridization rate in the presence of digoxin also nearly doubles (Figure 2C, green line $-t_{1 / 2}{ }^{+ \text {Digoxin }}=19 \mathrm{~min}$ ) and reaches a level near the hybridization rate of the Ctrl signaling strand, which lacks the digoxigenin moeity and therefore cannot bind the antibody (black line $\left.-t_{1 / 2}{ }^{\mathrm{Ctrl}}=12 \mathrm{~min}\right)$. Of note, the signal gain $\left(S^{\text {digoxin }} /\right.$ $\left.S^{\text {no_digoxin }}\right)$ is already optimal $(\sim 100 \%)$ only 5 min after adding the sample to the electrode (inset of Figure 2C). We also demonstrated the quantitative aspect of CeSHHA by interrogating samples with digoxin concentration ranging from $\mathrm{pM}$ to $\mu \mathrm{M}$. As expected we found that the sensor displays a typical 100 -fold dynamic range ${ }^{37}$ from $10 \mathrm{nM}$ to $1 \mu \mathrm{M}$ (Figure 2D).

In order to highlight the universality of this sensor design, we also adapted CeSHHA for the quantitative detection of TNT (2,4,6-trinitrotoluene), a small nitroaromatic explosive that represents an important class of soil pollutants. ${ }^{38}$ To do so, we employed an anti-TNT antibody, which is also known to bind the analogue DNP (2,4-dinitrophenol) although with poorer affinity. ${ }^{39}$ Again, DNP was attached to our redox-label signaling DNA strand in order to promote optimal assay sensitivity toward TNT, the molecule of interest in our assay (see Materials and Methods in Supporting Information). We first determined the concentration of anti-TNT antibody that is required to saturate the signaling DNA strand $(100 \mathrm{nM})$ in a $100 \mu \mathrm{L}$ sample volume (350 $\mathrm{nM}$, see Figure S5). Using this concentration we find that the TNT sensor displays similar gain $(\sim 100 \%$ see Figure $2 \mathrm{E})$ and kinetics (Figure $2 \mathrm{~F} ; t_{1 / 2}{ }^{\text {No TNT }}=$ $33 \mathrm{~min}, t_{1 / 2}{ }^{\mathrm{TNT}}=18 \mathrm{~min}$ ) to those observed with the digoxin sensor. The TNT sensor also displays a typical 100-fold dynamic range, ${ }^{37}$ albeit at a slightly higher TNT concentration $\left(100 \mathrm{nM}\right.$ to $10 \mu \mathrm{M}$ ) (Figure $2 \mathrm{G} ; C_{50 \%}=650 \mathrm{nM}$ ), which is likely due to the higher concentration of TNT-antibody that was used in this assay in order to ensure that most DNP-labeled signaling strands are bound by the antibody. This $C_{50 \%}$ is consistent with the fact that $650 \mathrm{nM}$ of TNT should leave around $100 \mathrm{nM}$ of antibody binding sites available, which in return likely bind half of our DNP-labeled signaling strands (not 1:1 due to the poorer DNP-antibody affinity).

We can also program CeSHHA to detect different ranges of target concentration by simply increasing and decreasing the concentration of antibody employed in the assay (Figure 3). A smaller concentration of digoxin antibody (thus a higher antiserum dilution of 1/66), for example, requires a lower concentration of free digoxin in order to have half of its binding sites sequestered (Figure 3B, blue line; $C_{50 \%}=5 \mathrm{nM}$ ). When increasing the antibody concentration in the assay to $1 / 20$ or to 1/6.6 antiserum dilutions, the dose-response curves obtained are right-shifted to higher digoxin concentrations since more digoxin is required to saturate the antibody binding sites (Figure 3B, $C_{50 \%}=35 \mathrm{nM}$ and $170 \mathrm{nM}$, respectively). Also, as expected with regard to the assay background, the higher the antibody concentration in the assay, the lower the measured current is in the absence of target (Figure 3A) or at low target concentration (Figure $3 \mathrm{~B}$ ). A trade-off is therefore required in 

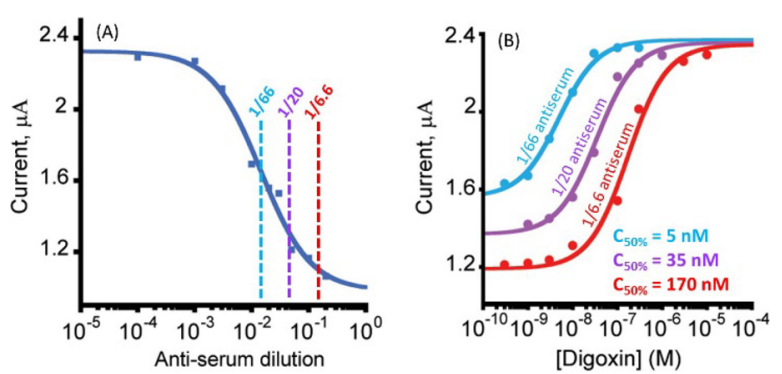

Figure 3. Tuning the dynamic range of CeSHHA. (A) Background signal of the sensor in the absence of digoxin when using different dilutions of anti-digoxin antiserum ${ }^{29}$ shows a $C_{50 \%}$ at a $1 / 66$ dilution. (B) Dose-response curve of the digoxin sensor using $1 / 66,1 / 20$, and 1/6.6 dilutions of anti-digoxin antibody. Employing larger antibody concentration proportionally shifts the dynamic range to higher concentration of digoxin $(1-30 \mathrm{nM}, 2-300 \mathrm{nM}$, and $20-2000 \mathrm{nM}$, respectively) while reducing the background signal of the sensor. Sample volume $=100 \mu \mathrm{L}$.

order to optimize the detection limit of the sensor (optimal here using a 66-fold dilution: $\mathrm{LOD}=1 \mathrm{nM}$ ). While a low concentration of antibody renders the sensor more sensitive to lower target concentrations, it also increases the sensor background in the absence of the analyte thus reducing the overall gain of the sensor (Figure S6).

One of the most significant advantages of CeSHHA is that it is one of the few universal sensor mechanisms that enable the selective detection of small molecules directly in complex samples, such as whole blood (see refs 28 and 40 for other examples). To demonstrate this, we compared the performance of the digoxin sensor in buffer and whole blood samples and found that the gains and kinetics of the assay are relatively similar in either matrix (Figure S7 and Figure 4). Using a low
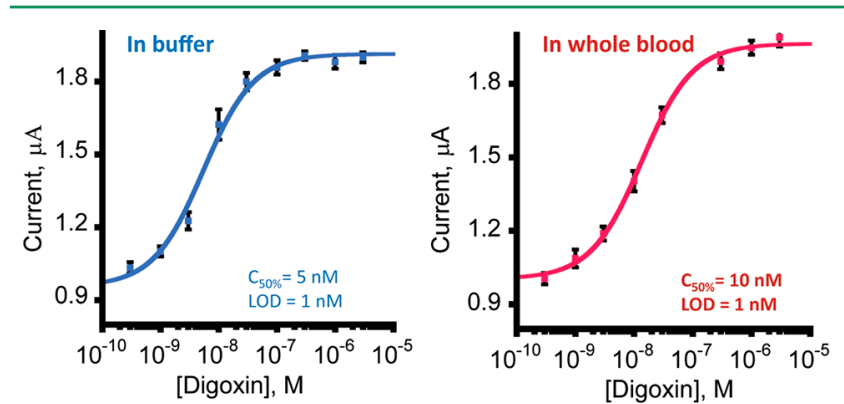

Figure 4. Quantitative measurement of digoxin in whole blood using CeSHHA. A dose-response curve of digoxin (A) in buffer displays a $\mathrm{C}_{50 \%}$ of $5 \mathrm{nM}$ and a 100 -fold linear dynamic range and (B) in whole blood displays a $C_{50 \%}$ of $10 \mathrm{nM}$ and a 100 -fold linear range. The 100 $\mu \mathrm{L}$ whole blood samples were first spiked with a specific concentration of digoxin. A 1/66 dilution of antiserum was then added to the sample followed by a $5 \mathrm{~min}$ incubation. The $100 \mathrm{nM}$ signaling strand was then added to the sample $10 \mathrm{~min}$ before sample-loading and initiating the acquisition. The signal was acquired after $30 \mathrm{~min}$ of acquisition. The error bars represent the typical standard error obtained when working with three electrodes, and are dominated by interelectrode variability.

antibody concentration $(1 / 66)$, we found that the dynamic range of this sensor ranges from $1 \mathrm{nM}$ to $30 \mathrm{nM}$ of digoxin concentration in both buffer and whole blood (Figure 4A and $\mathrm{B}$, respectively). These results confirm that CeSHHA can detect clinically relevant concentrations of digoxin directly in whole blood in less than $20 \mathrm{~min}$ ( $15 \mathrm{~min}$ incubation and $5 \mathrm{~min}$ hybridization/acquiring time) through a simple "one-pot" reaction. CeSHHA compares advantageously well with other recently reported sensors for the detection of digoxin. For example, Bagheri et al. have recently reported an aptamer-based electrochemical digoxin sensor that works in urine or blood plasma but with a dynamic range $(26-260 \mathrm{pM})$ that is outside of the clinically relevant dixogin concentrations. ${ }^{41} \mathrm{We}$ also challenged the TNT sensor directly in soil samples using $1 / 2$ dilution of soil in buffer. We find that the TNT sensor displays a similar quantitative performance $\left(C_{50 \%}\right.$ of $\left.650 \mathrm{nM}\right)$ even when employed directly in the soil sample (Figure S8), which is sensitive enough to detect the maximal accepted levels of TNT in soils $\left(17.2 \mathrm{mg} \mathrm{kg}{ }^{-1}\right){ }^{19}$ Taken together, these results (detection of small molecules directly in whole blood or soil) demonstrate that CeSHHA is robust and selective enough to achieve small analyte detection directly in blood or soil samples without requiring cumbersome (and expensive) centrifugation (e.g., to obtain serum and plasma), separation, purification, or washing steps as most assays or biosensors typically do.

Here, we have described a potentially universal DNA-based immunoassay for the detection of small molecules that enables signal-on, quantitative detection directly in complex sample, such as whole blood, in less than $20 \mathrm{~min}$. This antibody/DNA based immunoassay, called CeSHHA, employs the high specificity both of DNA hybridization and of antibody recognition to do so. We have demonstrated the adaptation of this assay for the rapid detection $(<20 \mathrm{~min})$ of (1) digoxin directly in whole blood within low nanomolar concentrations, and (2) TNT directly in soil media. CeSHHA could, in principle, be adapted for the detection of any small molecule for which a specific large recognition element (e.g., antibody or protein) is available. Another important advantage of CeSHHA is that it also enables optimization of the sensor dynamic range over a wide range of concentrations ( $>100$-fold) by increasing or decreasing the concentration of antibody. In principle, CeSHHA is also capable of enabling multiplexed detection of numerous small target molecules, by taking advantage of the unique ability of DNA to create numerous specific capturingsignaling pairs. ${ }^{29}$ CeSHHA also displays significant advantages compared to other universal DNA-based sensors such as the EDNA scaffold platform. ${ }^{39,40,42}$ In addition to being robust enough to enable detection directly in whole blood, CeSHHA also provides a 20 -fold enhancement in the electrochemical current due to its ability to work at high surface density.

Given all these advantages, CeSHHA represents a potentially generalizable sensing platform to enable the rapid, inexpensive, and quantitative detection of potentially multiple molecules directly in whole blood. CeSHHA therefore represents one of the few sensing mechanisms insensitive to nonspecific adsorption of proteins on the sensor surface. With its simplicity of use (two steps all performed directly in a drop of blood), we believe that this assay possesses all the characteristics to be readily applied for applications ranging from POC diagnosis of numerous diseases to real-time monitoring of disease treatment.

\section{ASSOCIATED CONTENT}

\section{Supporting Information}

The Supporting Information is available free of charge on the ACS Publications website at DOI: 10.1021/acssensors.7b00176.

Materials and methods, optimization of capturing strand density and signaling strand concentration, dose 
response curves of anti-digoxin antiserum and anti-TNT antibody, optimization of detection limit, CeSHHA response in whole blood and soil (PDF)

\section{AUTHOR INFORMATION}

\section{Corresponding Author}

*E-mail: a.vallee-belisle@umontreal.ca.

\section{ORCID}

Francesco Ricci: 0000-0003-4941-8646

Shana O. Kelley: 0000-0003-3360-5359

Alexis Vallée-Bélisle: 0000-0002-5009-7715

Notes

The authors declare no competing financial interest.

\section{ACKNOWLEDGMENTS}

This work was supported by a NSERC discovery grant (RGPIN-2014-06403 to A.V.-B), the European Research Council (ERC, 336493 to F.R.), and the EU-IRSES grant "Chimera" (F.R and A.V.-B.). S.S.M. is a NSERC postdoctoral fellow. A.V.-B. is a CRC in Bioengineering and Bionanotechnology, Tier II. We thank PROTEO, the CCVC, and Liliana Pedro for discussion.

\section{REFERENCES}

(1) Yager, P.; Domingo, G. J.; Gerdes, J. Point-of-Care Diagnostics for Global Health. Annu. Rev. Biomed. Eng. 2008, 10, 107-44.

(2) Yetisen, A. K.; Akram, M. S.; Lowe, C. R. Paper-Based Microfluidic Point-of-Care Diagnostic Devices. Lab Chip 2013, 13 (12), 2210-51.

(3) Wang, J. Electrochemical biosensors: towards point-of-care cancer diagnostics. Biosens. Bioelectron. 2006, 21 (10), 1887-92.

(4) Kumar, A. A.; Hennek, J. W.; Smith, B. S.; Kumar, S.; Beattie, P.; Jain, S.; Rolland, J. P.; Stossel, T. P.; Chunda-Liyoka, C.; Whitesides, G. M. From the Bench to the Field in Low-Cost Diagnostics:TwoCase Studies. Angew. Chem., Int. Ed. 2015, 54, 5836-5853.

(5) Bissonnette, L.; Bergeron, M. G. Diagnosing infections-current and anticipated technologies for point-of-care diagnostics and homebased testing. Clin. Microbiol. Infect. 2010, 16 (8), 1044-53.

(6) Gardiner, C.; Williams, K.; Mackie, I. J.; Machin, S. J.; Cohen, H. Patient self-testing is a reliable and acceptable alternative to laboratory INR monitoring. Br. J. Haematol. 2005, 128 (2), 242-7.

(7) Clarke, S. F.; Foster, J. R. A history of blood glucose meters and their role in self-monitoring of diabetes mellitus. British Journal of Biomedical Science 2012, 69 (2), 83-93.

(8) Luong, J. H.; Male, K. B.; Glennon, J. D. Biosensor technology: technology push versus market pull. Biotechnol. Adv. 2008, 26 (5), 492-500.

(9) Lan, T.; Zhang, J. J.; Lu, Y. Transforming the blood glucose meter into a general healthcare meter for in vitro diagnostics in mobile health. Biotechnol. Adv. 2016, 34 (3), 331-341.

(10) Ziegler, S.; Pries, V.; Hedberg, C.; Waldmann, H. Target identification for small bioactive molecules: finding the needle in the haystack. Angew. Chem., Int. Ed. 2013, 52 (10), 2744-92.

(11) Bauer, A.; Stockwell, B. Neurobiological applications of small molecule screening. Chem. Rev. 2008, 108 (5), 1774-86.

(12) Plowright, A. T.; Engkvist, O.; Gill, A.; Knerr, L.; Wang, Q. D. Heart regeneration: opportunities and challenges for drug discovery with novel chemical and therapeutic methods or agents. Angew. Chem., Int. Ed. 2014, 53 (16), 4056-75.

(13) Iorio, M. V.; Croce, C. M. MicroRNA dysregulation in cancer: diagnostics, monitoring and therapeutics. A comprehensive review. EMBO Mol. Med. 2012, 4 (3), 143-59.

(14) Liu, J.; Bu, J.; Bu, W.; Zhang, S.; Pan, L.; Fan, W.; Chen, F.; Zhou, L.; Peng, W.; Zhao, K.; Du, J.; Shi, J. Real-time in vivo quantitative monitoring of drug release by dual-mode magnetic resonance and upconverted luminescence imaging. Angew. Chem., Int. Ed. 2014, 53 (18), 4551-5.

(15) Khan, A.; Khan, A. A.; Asiri, A. M.; Rub, M. A.; Azum, N.; Rahman, M. M.; Khan, S. B.; Ghani, S. A. A new trend on biosensor for neurotransmitter choline/acetylcholine-an overview. Appl. Biochem. Biotechnol. 2013, 169 (6), 1927-39.

(16) Krol, S.; Macrez, R.; Docagne, F.; Defer, G.; Laurent, S.; Rahman, M.; Hajipour, M. J.; Kehoe, P. G.; Mahmoudi, M. Therapeutic benefits from nanoparticles: the potential significance of nanoscience in diseases with compromise to the blood brain barrier. Chem. Rev. 2013, 113 (3), 1877-903.

(17) Su, Z.; Zhang, Y.; Gendron, T. F.; Bauer, P. O.; Chew, J.; Yang, W. Y.; Fostvedt, E.; Jansen-West, K.; Belzil, V. V.; Desaro, P.; Johnston, A.; Overstreet, K.; Oh, S. Y.; Todd, P. K.; Berry, J. D.; Cudkowicz, M. E.; Boeve, B. F.; Dickson, D.; Floeter, M. K.; Traynor, B. J.; Morelli, C.; Ratti, A.; Silani, V.; Rademakers, R.; Brown, R. H.; Rothstein, J. D.; Boylan, K. B.; Petrucelli, L.; Disney, M. D. Discovery of a biomarker and lead small molecules to target r(GGGGCC)associated defects in c9FTD/ALS. Neuron 2014, 83 (5), 1043-50.

(18) Costa, J.; de Carvalho, M. Emerging molecular biomarker targets for amyotrophic lateral sclerosis. Clin. Chim. Acta 2016, 455, $7-14$.

(19) Zhang, J. J.; Xiang, Y.; Wang, M.; Basu, A.; Lu, Y. DoseDependent Response of Personal Glucose Meters to Nicotinamide Coenzymes: Applications to Point-of-Care Diagnostics of Many NonGlucose Targets in a Single Step. Angew. Chem., Int. Ed. 2016, 55 (2), $732-736$.

(20) Mahshid, S. S.; Mahshid, S.; Dolati, A.; Ghorbani, M.; Yang, L.; Luo, S.; Cai, Q. Electrodeposition and electrocatalytic properties of $\mathrm{Pt} / \mathrm{Ni}-\mathrm{Co}$ nanowires for non-enzymatic glucose detection. J. Alloys Compd. 2013, 554, 169-176.

(21) Mahshid, S.; Li, C.; Mahshid, S. S.; Askari, M.; Dolati, A.; Yang, L.; Luo, S.; Cai, Q. Sensitive determination of dopamine in the presence of uric acid and ascorbic acid using $\mathrm{TiO} 2$ nanotubes modified with Pd, Pt and Au nanoparticles. Analyst 2011, 136 (11), 2322-9.

(22) Shrivastava, S.; Jadon, N.; Jain, R. Next-generation polymer nanocomposite-based electrochemical sensors and biosensors: A review. TrAC, Trends Anal. Chem. 2016, 82, 55-67.

(23) Si, P.; Huang, Y. J.; Wang, T. H.; Ma, J. M. Nanomaterials for electrochemical non-enzymatic glucose biosensors. RSC Adv. 2013, 3 (11), 3487-3502.

(24) Miao, Y. Q.; Ouyang, L.; Zhou, S. L.; Xu, L.; Yang, Z. Y.; Xiao, M. S.; Ouyang, R. Z. Electrocatalysis and electroanalysis of nickel, its oxides, hydroxides and oxyhydroxides toward small molecules. Biosens. Bioelectron. 2014, 53, 428-439.

(25) Vallée-Bélisle, A.; Plaxco, K. W. Structure-switching biosensors: inspired by nature. Curr. Opin. Struct. Biol. 2010, 20 (4), 518-26.

(26) Labib, M.; Sargent, E. H.; Kelley, S. O. Electrochemical Methods for the analysis of clinically relevant biomolecules. Chem. Rev. 2016, 116 (16), 9001-90.

(27) Frost, M. C.; Meyerhoff, M. E. Real-time monitoring of crititical care analytes in the bloodstream with chemical sensors: progress and challenges. Annu. Rev. Anal. Chem. 2015, 8, 171-92.

(28) Li, H.; Arroyo-Curras, N.; Kang, D.; Ricci, F.; Plaxco, K. W. Dual-reporter drift correction to enhance the performance of electrochemical aptamer-based sensors in whole blood. J. Am. Chem. Soc. 2016, 138 (49), 15809-12.

(29) Mahshid, S. S.; Camire, S.; Ricci, F.; Vallee-Belisle, A. A Highly Selective Electrochemical DNA-Based Sensor That Employs Steric Hindrance Effects to Detect Proteins Directly in Whole Blood. J. Am. Chem. Soc. 2015, 137 (50), 15596-9.

(30) Van Weemen, B. K.; Schuurs, A. H. W. M. Immunoassay Using Antigen-Enzyme Conjugates. FEBS Lett. 1971, 15 (3), 232-236.

(31) Engvall, E.; Perlmann, P. Enzyme-Linked Immunosorbent Assay (ELISA). Quantitative Assay of Immunoglobulin G. Immunochemistry 1971, 8 (9), 871-4.

(32) Li, X.; Wang, Y.; Zhou, Q.; Yu, Y.; Chen, L.; Zheng, J. A sensitive method for digoxin determination using formate-adduct ion based on the effect of ionization enhancement in liquid chromato- 
graph-mass spectrometer. J. Chromatogr. B: Anal. Technol. Biomed. Life Sci. 2015, 978-979, 138-44.

(33) Lim, A. E.; Tate, J. R.; Clarke, D.; Norris, R. L.; Morris, R. G.; Martin, J. H. Clinical consequences of a miscalibrated digoxin immunoassay. Ther. Drug Monit. 2015, 37 (1), 104-9.

(34) Gheorghiade, M.; Adams, K. F., Jr.; Colucci, W. S. Digoxin in the management of cardiovascular disorders. Circulation 2004, 109 (24), 2959-64.

(35) Scalese, M. J.; Salvatore, D. J. Role of Digoxin in Atrial Fibrillation. J. Pharm. Pract 2016, DOI: 10.1177/0897190016642361.

(36) Kelley, S. O.; Barton, J. K.; Jackson, N. M.; Hill, M. G. Electrochemistry of methylene blue bound to a DNA-modifier electrode. Bioconjugate Chem. 1997, 8 (1), 31-7.

(37) Vallée-Bélisle, A.; Ricci, F.; Plaxco, K. W. Engineering Biosensors with Extended, Narrowed, or Arbitrarily Edited Dynamic Range. J. Am. Chem. Soc. 2012, 134 (6), 2876-9.

(38) Spain, J. C. Biodegradation of nitroaromatic compounds. Annu. Rev. Microbiol. 1995, 49, 523-55.

(39) Cash, K. J.; Ricci, F.; Plaxco, K. W. A general electrochemical method for label-free screening of protein-small molecule interactions. Chem. Commun. 2009, No. 41, 6222-4.

(40) Arroyo-Curras, N.; Somerson, J.; Vieira, P. A.; Ploense, K. L.; Kippin, T. E.; Plaxco, K. W. Real-time measurement of small molecules directly in awake, ambulatory animals. Proc. Natl. Acad. Sci. U. S. A. 2017, 114 (4), 645-50.

(41) Bagheri, H.; Talemi, R. P.; Afkhami, A. Gold nanoparticules deposited on fluorine-doped tin oxide surface as an effective platform for fabricating a highly sensitive and specific digoxin aptasensor. RSC Adv. 2015, 5 (5), 58491-8.

(42) Cash, K. J.; Ricci, F.; Plaxco, K. W. An electrochemical sensor for the detection of protein-small molecule interactions directly in serum and other complex matrices. J. Am. Chem. Soc. 2009, 131 (20), 6955-7. 